\title{
Variable uterine uptake of FDG in adenomyosis during concurrent chemoradiation therapy for cervical cancer
}

\author{
Jeong II Yu, MD¹, Seung Jae Huh, MD¹, Young II Kim, MD1', Tae-Joong Kim, MD², Byung Kwan Park, MD³ \\ Departments of ${ }^{1}$ Radiation Oncology, ${ }^{2}$ Obstetrics and Gynaecology, ${ }^{3}$ Radiology, Samsung Medical Center, \\ Sungkyunkwan University School of Medicine, Seoul, Korea
}

To avoid improper tumor volume contouring in radiation therapy (RT) and other invasive procedures, we report a case of uterine adenomyosis showing increased ${ }^{18} \mathrm{~F}$-fluorodeoxyglucose (FDG) uptake on positron emission tomography (PET)/computed tomography (CT) mimicking malignant tumor in a 44-year-old woman during concurrent chemoradiation therapy (CCRT) for uterine cervical cancer. The adenomyosis was not associated with her menstrual cycle or with normal endometrium uptake, and it resolved one month after completion of RT. This case indicates that uterine adenomyosis in a premenopausal woman may show false positive uptake of ${ }^{18} \mathrm{FDG}-\mathrm{PET} / \mathrm{CT}$ associated with CCRT.

Keywords: Positron emission tomography/computed tomography, Concurrent chemoradiation therapy, Adenomyosis

\section{Introduction}

Positron emission tomography (PET)/computed tomography (CT) with ${ }^{18}$ F-fluorodeoxyglucose (FDG) is used extensively for staging and monitoring in patients with uterine cervical cancer [1]. Because of high glucose metabolism, FDG generally accumulates in malignant lesions. FDG uptake, however, is not specific for cancer cells and, therefore, sometimes results in false-positive conclusions for benign inflammatory lesions [2]. We report a case of uterine adenomyosis showing increased ${ }^{18} \mathrm{FDG}$ uptake on PET/CT mimicking malignant tumor in a 44-year-old woman during concurrent chemoradiation therapy (CCRT) for uterine cervical cancer. However, the ${ }^{18}$ FDG uptake was completely resolved one month after completion of RT. Our case indicates that uterine adenomyosis in a premenopausal woman may result in false positive ${ }^{18} \mathrm{FDG}-\mathrm{PET} /$
$\mathrm{CT}$ findings. To our knowledge, false positive uterine ${ }^{18} \mathrm{FDG}$ uptake in adenomyosis during CCRT for cervical cancer has not been previously described.

\section{Case Report}

A 44-year-old woman presented with a two-week history of painless abnormal vaginal bleeding and was referred to the gynecology department in August 2010. Upon pelvic examination, a 4-cm exophytic, cancerous lesion was identified in the uterine cervix that extended into the cervical canal. The right side of the parametrium was fixed. Punch biopsies were collected, and invasive squamous cell carcinoma of the cervix confirmed. There was no evidence of distant metastasis at that time. International Federation of Gynecology and Obstetrics (FIGO) stage IIB cervical cancer was diagnosed.

Received 25 May 2011, Accepted 10 June 2011.

Correspondence: Seung Jae Huh, MD, Department of Radiation Oncology, Samsung Medical Center, Sungkyunkwan University School of Medicine, 50 Irwon-dong, Gangnam-gu, Seoul 135-710, Korea. Tel: +82-2-3410-2605, Fax: +82-2-34102601, E-mail: sj5201.huh@samsung.com

(C) This is an Open Access article distributed under the terms of the Creative Commons Attribution Non-Commercial License (http://creativecommons.org/ licenses/by-nc/3.0/) which permits unrestricted non-commercial use, distribution, and reproduction in any medium, provided the original work is properly cited. www.e-roj.org 


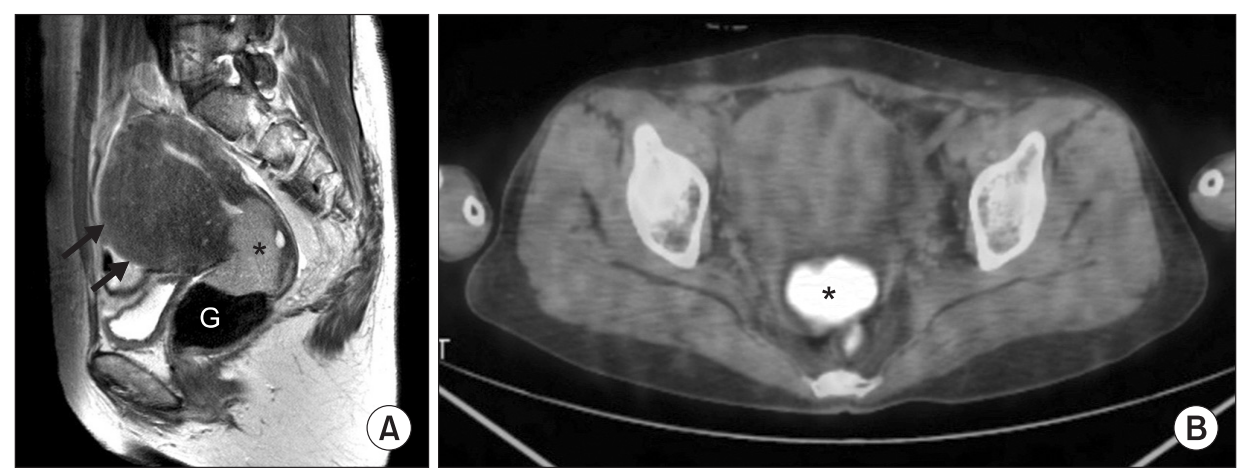

Fig. 1. (A) T2-weighted sagittal magnetic resonance (MR) image obtained prior to concurrent chemoradiation therapy shows hyper intense cervical cancer (asterisk) in the cervical canal. Black arrows indicate thickened anterior myometrium which contains multiple hyperintense foci, suggesting adenomyosis. Gauze $(\mathrm{G})$ is packed in the vaginal canal. (B) Fusion axial ${ }^{18}$ F-fluorodeoxyglucosepositron emission tomography/computed tomography image one day after MR imaging of (A) shows cervical cancer (asterisk) with hypermetabolic glucose.

Magnetic resonance imaging (MRI) showed a 4.5-cm cervical tumor with direct invasion of the right parametrium and upper vagina. Multiple lymph node enlargements were also identified along the right external iliac and right retrocaval chain. In the uterus, multiple uterine myomas and an adenomyosis were noted (Fig. 1A). ${ }^{18}$ FDG-PET/CT examination was performed, demonstrating significant uptake in the uterine cervix mass, right external iliac and retrocaval lymph nodes (Fig. 1B).

The patient underwent CT scans for radiation therapy (RT) planning. The gross target volumes were defined as the lesions noted on the MRI and ${ }^{18} \mathrm{FDG}-\mathrm{PET} / \mathrm{CT}$. The clinical target volumes included the entire pelvis and the para-aortic area up to the upper body of the first lumbar spine. The major tumor and whole pelvis were treated over six weeks with 28 fractions of 50.4 Gy with anteroposterior/posteroanterior RT fields of 15-MV photons.

The regimen for CCRT consisted of six cycles of 5 -fluorouracil $\left(1,000 \mathrm{mg} / \mathrm{m}^{2}\right.$ in a continuous infusion over 96 hours $)$ and cisplatin $\left(60 \mathrm{mg} / \mathrm{m}^{2}\right)$ at three-week intervals. On interim $\mathrm{MRI}$ and $\mathrm{PET} / \mathrm{CT}$ imaging for intracavitary radiotherapy (ICR) planning after the 23rd fraction of 4,140 cGy external radiotherapy, tumor size and ${ }^{18} \mathrm{FDG}$ uptake in the cervix were markedly decreased. However, a new region of abnormal uptake appeared around a pre-existing adenomyosis in the anterior body of the uterus (Fig. 2).

After external radiotherapy, PET/CT-guided high-dose ICR using an iridium-192 source was carried out with a reference tumor prescription of $24 \mathrm{~Gy}$, three times a week in six fractions. One month after completion of RT, follow-up imaging was performed. There was no evidence of malignancy on either MRI or ${ }^{18}$ FDG-PET/CT. In addition, ${ }^{18}$ FDG uptake in the uterine anterior body was completely resolved (Fig. 3). The patient was premenopausal; however, menstruation did not occur after CCRT. Delayed toxicity related to RT was also reported.

\section{Discussion and Conclusion}

Adenomyosis is a common non-neoplastic gynecologic disease characterized by excessive migration of the endometrial glands and stroma from the basal layer of the endometrium into the myometrium [3]. The ectopic endometrium may be localized or diffusely distributed throughout the myometrium, leading to the formation of adenomyomas [4]. The diagnosis is usually based on histological findings in surgical specimens. MRI is a widely accepted method for achieving a valid pre-surgical diagnosis [5]. Some research has reported that postmenopausal patients with no known gynecologic malignancy had very mild FDG uptake [6]. Therefore, in postmenopausal women, all increased endometrial uptake is of clinical significance, and further investigation of the uterine pathology is advised.

However, in premenopausal women, endometrial uptake is related to the menstrual cycle and is highest during menses, followed by the level in the ovulation phase [5]. Endometrial FDG uptake during menstruation may be related to the peristaltic movements of the subendometrial myometrium that help to discharge menstrual blood [5]. The endometrial uptake in premenopausal patients using oral contraceptives resembles that in non-ovulating, non-menstruating premenopausal subjects [6], which is likely secondary to the effects of oral contraceptives including suppression of glands and atrophic introduction of the endometrium.

In our case, endometrial tissue in the myometrium showed 

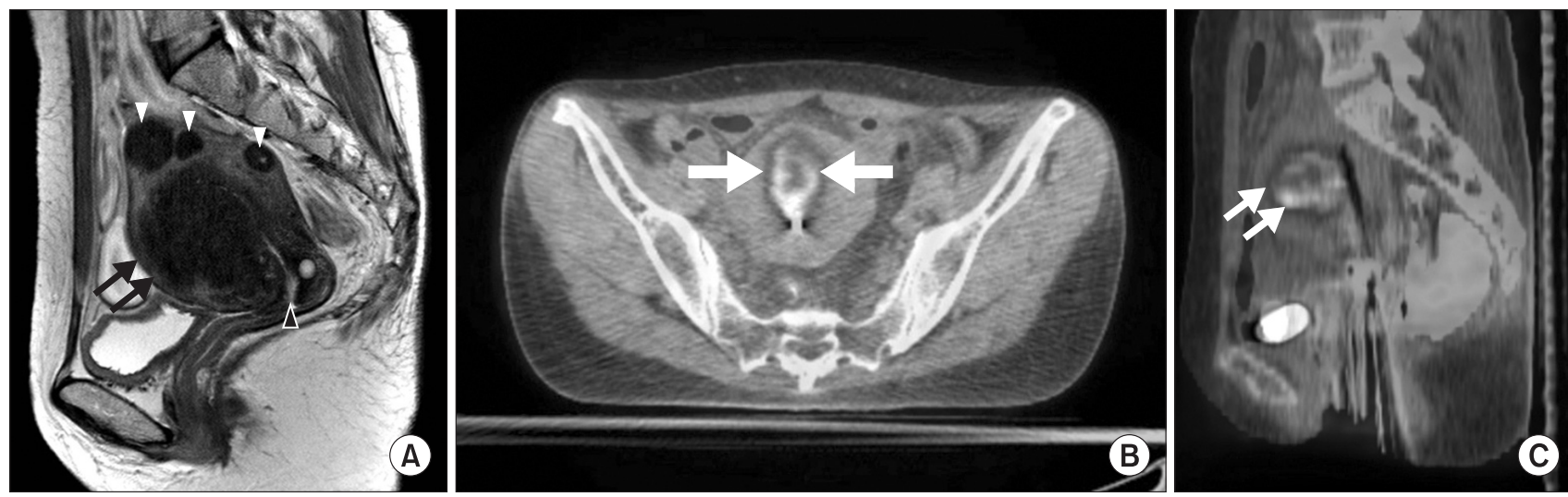

Fig. 2. (A) T2-weighted sagittal magnetic resonance (MR) image obtained during concurrent chemoradiation therapy (CCRT; external radiotherapy 4,140 cGy/23 fraction) shows no residual cervical cancer within the cervical canal (black arrowhead). The adenomyosis (black arrows) is better demarcated than that in Fig. 1A. Note the multiple small myomas (white arrowheads) in the myometrium. Fusion axial (B) and sagittal (C) ${ }^{18} \mathrm{~F}$-fluorodeoxyglucose-positron emission tomography (PET)/computed tomography (CT) images one day after MR imaging of Fig. 2A for PET/CT image-guided brachytherapy with insertion of intrauterine tandem applicator show a hypermetabolic area (white arrows) that corresponds to the adenomyosis.
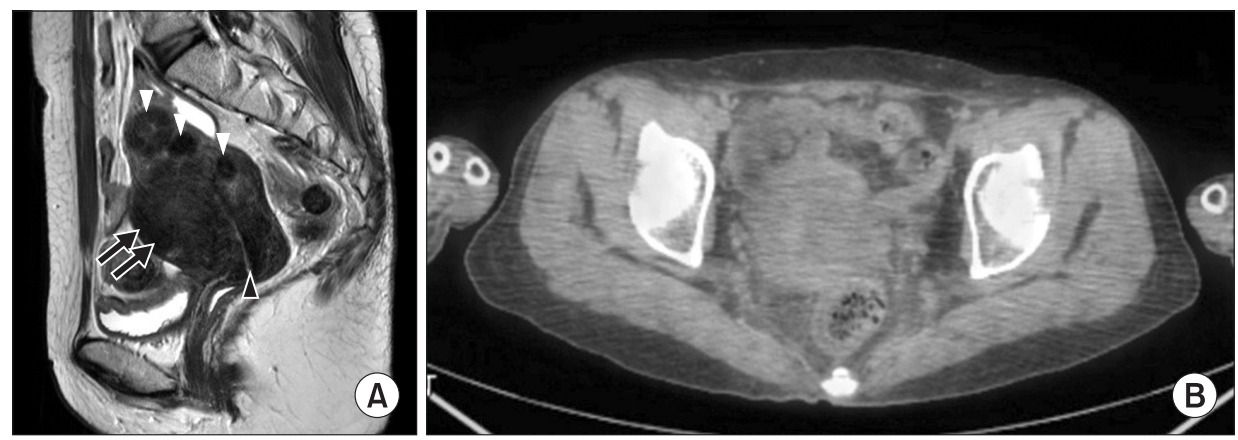

Fig. 3. (A) T2-weighted sagittal magnetic resonance (MR) image obtained one month after completion of radiation therapy shows no residual or recurrent cervical cancer in the cervical canal (black arrowhead). The adenomyosis (black arrows) is poorly demarcated compared to that in Fig. 2A. White arrowheads indicate intramural myomas. (B) Fusion axial ${ }^{18} \mathrm{~F}$-fluorodeoxyglucose-positron emission tomography/computed tomography image on the same day as MR imaging (A) shows no hypermetabolic area in the adenomyosis.

new ${ }^{18}$ FDG uptake (standardized uptake values, 5.1) during CCRT that was not detected during the staging examination. The possibility of physiologic ${ }^{18} \mathrm{FDG}$ endometrial uptake was suggested, but her normal endometrium did not exhibit significant uptake. Adenomyosis generally shows mild FDG uptake in premenopausal women, with increased uptake during menstruation and ovulation [7].

High uptake of ${ }^{18} \mathrm{FDG}$ PET/CT during CCRT (external radiotherapy 4,140 cGy/23 fraction) in this case was assumed to have no association with the menstrual cycle as the patient reported no menstruation after the initiation of CCRT. Cole previously reported that permanent amenorrhea was induced after a single pelvic radiation dose of $4.5 \mathrm{~Gy}$ in 90\% (70/78) of women aged 40 to 44 years, 96\% (71/78) of women aged 45 to 49 years, and $100 \%(25 / 25)$ of women older than 49 years [8]. Considering that radiation might cause an immunologic reaction at the irradiated site, in addition to direct injury [8], this temporary abnormal uptake during CCRT may be related to certain inflammatory responses of adenomyosis due to radiation damage.

The utilization of ${ }^{18} \mathrm{FDG}-\mathrm{PET} / \mathrm{CT}$ in the staging and assessment of cervical malignancies has been rapidly increasing in recent years. However, false-positive results have been reported with ${ }^{18} \mathrm{FDG}-\mathrm{PET} / \mathrm{CT}$ in the uterus and are thought to be the results of physiologic and benign causes. Moreover, in patients with known cervical cancer, endometrial uptake may be increased even though no endometrial invasion or involvement is actually present [6]. Therefore, proper ${ }^{18} \mathrm{FDG}-\mathrm{PET} / \mathrm{CT}$ image 
interpretation requires knowledge of physiologic and normal biodistributions of ${ }^{18} \mathrm{FDG}$ throughout the body.

As exemplified in this case, the abnormalities seen on ${ }^{18} \mathrm{FDG}$ $\mathrm{PET} / \mathrm{CT}$ must be compared to anatomic imaging results such as those of MRI, for further characterization as likely malignant or benign. We report this case because failure to recognize this kind of potential focal uptake on an ${ }^{18} \mathrm{FDG}-\mathrm{PET} / \mathrm{CT}$ scan may lead to an erroneous interpretation and improper tumor volume contouring for image-guided radiation therapy.

\section{Conflict of Interest}

No potential conflict of interest relevant to this article was reported.

\section{References}

1. Grigsby PW. The prognostic value of PET and PET/CT in cervical cancer. Cancer Imaging 2008:8:146-55.
2. Liu Y, Ghesani NV, Zuckier LS. Physiology and pathophysiology of incidental findings detected on FDG-PET scintigraphy. Semin Nucl Med 2010;40:294-315.

3. Exacoustos $C$, Brienza AL, Di Giovanni $A$, et al. Adenomyosis: three dimensional (3D) sonographic findings of the junctional zone and correlation to histology. Ultrasound Obstet Gynecol 2011;37:471-9.

4. Graham KJ, Hulst FA, Vogelnest L, Fraser IS, Shilton CM. Uterine adenomyosis in an orang-utan (Pongo abelii) pygmaeus). Aust Vet J 2009;87:66-9.

5. Fujiwara $T$, Togashi K, Yamaoka $T$, et al. Kinematics of the uterus: cine mode MR imaging. Radiographics 2004;24:e19.

6. Lerman H, Metser U, Grisaru D, Fishman A, Lievshitz G, Even-Sapir E. Normal and abnormal 18F-FDG endometrial and ovarian uptake in pre- and postmenopausal patients: assessment by PET/CT. J Nucl Med 2004;45:266-71.

7. Kitajima K, Murakami K, Kaji Y, Sugimura K. Spectrum of FDG PET/CT findings of uterine tumors. AJR Am J Roentgenol 2010;195:737-43.

8. Signorile PG, Baldi A. Endometriosis: new concepts in the pathogenesis. Int J Biochem Cell Biol 2010;42:778-80. 